Review

\title{
Quantum Local Symmetry of the $D$-Dimensional Non-Linear Sigma Model: A Functional Approach
}

\author{
Andrea Quadri ${ }^{1,2}$ \\ ${ }^{1}$ Istituto Nazionale di Fisica Nucleare (INFN), Sezione di Milano, via Celoria 16, I-20133 Milano, \\ Italy; E-Mail: andrea.quadri@mi.infn.it; Tel.: +39-2-5031-7287; Fax: +39-2-5031-7480 \\ ${ }^{2}$ Dipartimento di Fisica, Università di Milano, via Celoria 16, I-20133 Milano, Italy
}

Received: 27 February 2014; in revised form: 31 March 2014 / Accepted: 11 April 2014 /

Published: 17 April 2014

\begin{abstract}
We summarize recent progress on the symmetric subtraction of the Non-Linear Sigma Model in $D$ dimensions, based on the validity of a certain Local Functional Equation (LFE) encoding the invariance of the SU(2) Haar measure under local left transformations. The deformation of the classical non-linearly realized symmetry at the quantum level is analyzed by cohomological tools. It is shown that all the divergences of the one-particle irreducible (1-PI) amplitudes (both on-shell and off-shell) can be classified according to the solutions of the LFE. Applications to the non-linearly realized Yang-Mills theory and to the electroweak theory, which is directly relevant to the model-independent analysis of LHC data, are briefly addressed.
\end{abstract}

Keywords: Non-Linear Sigma Model; quantum symmetries; renormalization; Becchi-Rouet-Stora-Tyutin (BRST)

\section{Introduction}

The purpose of this paper is to provide an introduction to the recent advances in the study of the renormalization properties of the SU(2) Non-Linear Sigma Model (NLSM) and of the quantum deformation of the underlying non-linearly realized classical SU(2) local symmetry. The results reviewed here are based mainly on References [1-19].

The linear sigma model was originally proposed a long time ago in [20] in the context of elementary particle physics. In this model the pseudoscalar pion fields $\vec{\phi}$ form a chiral multiplet together with a 
scalar field $\sigma$, with $(\sigma, \vec{\phi})$ transforming linearly as a vector under $\mathrm{O}(4) \sim \mathrm{SU}(2) \times \mathrm{SU}(2) / \mathrm{Z}_{2}$. If one considers instead the model on the manifold defined by

$$
\sigma^{2}+\vec{\phi}^{2}=f_{\pi}^{2}, \quad \sigma>0
$$

one obtains a theory where the chiral group $\mathrm{SO}(4) \sim \mathrm{SU}(2) \times \mathrm{SU}(2)$ (with $\mathrm{SO}(4)$ selected by the positivity condition on $\sigma$ ) is spontaneously broken down to the isotopic spin group $\mathrm{SU}(2)$. The composite field $\sigma$ has a non-vanishing expectation value $f_{\pi}$ (to be identified with the pion decay constant), while the pions are massless. Despite the fact that this is only an approximate description (since in reality the pions are massive and chiral $\mathrm{SU}(2) \times \mathrm{SU}(2)$ is not exact, even before being spontaneously broken), the approach turned out to be phenomenologically quite successful and paved the way to the systematic use of effective field theories as a low energy expansion.

The first step in this direction was to obtain a phenomenological lagrangian directly, by making use of a pion field with non-linear transformation properties dictated by chiral symmetry from the beginning. After the seminal work of Reference [21] for the chiral $\mathrm{SU}(2) \times \mathrm{SU}(2)$ group, non-linearly realized symmetries were soon generalized to arbitrary groups in [22,23] and have since then become a very popular tool [24].

Modern applications involve, e.g., Chiral Perturbation Theory [25-28], low energy electroweak theories [29] as well as gravity [30].

Effective field theories usually exhibit an infinite number of interaction terms, that can be organized according to the increasing number of derivatives. By dimensional arguments, the interaction terms must then be suppressed by some large mass scale $M$ (so that one expects that the theory is reliable at energies well below M) (For a modern introduction to the problem, see e.g., [31]). In the spirit of the phenomenological lagrangians, the tree-level effective action is used to compute physical quantities up to a given order in the momentum expansion. Only a finite number of derivative interaction vertices contribute to that order, thus allowing to express the physical observables one is interested in through a finite number of parameters (to be eventually fixed by comparison with experimental data). Then the theory can be used to make predictions at the given order of accuracy in the low-energy expansion.

The problem of the mathematically consistent evaluation of quantum corrections in this class of models has a very long history. On general grounds, the derivative couplings tend to worsen the ultraviolet (UV) behavior of the theory, since UV divergent contributions arise in the Feynman amplitudes that cannot be compensated by a multiplicative renormalization of the fields and a redefinition of the mass parameters and the coupling constants in the classical action (truncated at some given order in the momentum expansion). Under these circumstances, one says that the theory is non-renormalizable (A compact introduction to renormalization theory is given in [32]).

It should be stressed that the key point here is the instability of the classical action: no matter how many terms are kept in the derivative expansion of the tree-level action, there exists a sufficiently high loop order where UV divergences appear that cannot be reabsorbed into the classical action. On the other hand, if in a non-anomalous and non-renormalizable gauge theory one allows for infinitely many terms in the classical action (all those compatible with the symmetries of the theory), then UV divergences can indeed be reabsorbed by preserving the Batalin-Vilkovisky master equation [33] and the model is said to be renormalizable in the modern sense [34]. 
Sometimes symmetries are so powerful in constraining the UV divergences that the non-linear theory proves to be indeed renormalizable (although not by power-counting), like for instance the NLSM in two dimensions [35,36] (For a more recent introduction to the subject, see e.g., [37]).

In four dimensions the situation is much less favorable. It has been found many years ago that already at one loop level in the four-dimensional NLSM there exists an infinite number of one-particle irreducible (1-PI) divergent pion amplitudes. Many attempts were then made in the literature in order to classify such divergent terms. Global SU(2) chiral symmetry is not preserved already at one loop level [38-40]. Moreover it turns out that some of the non-symmetric terms can be reabsorbed by a redefinition of the fields [40-43], however in the off-shell four-point $\phi_{a}$ amplitudes some divergent parts arise that cannot be reabsorbed by field redefinitions unless derivatives are allowed [40]. These technical difficulties prevented such attempts to evolve into a mathematically consistent subtraction procedure.

More recently it has been pointed out [1] that one can get the full control on the ultraviolet divergences of the $\phi$ 's-amplitudes by exploiting the constraints stemming from the presence of a certain local symmetry, associated with the introduction of a SU(2) background field connection into the theory. This symmetry in encoded in functional form in the so-called Local Functional Equation (LFE) [1]. It turns out that the fundamental divergent amplitudes are not those associated with the quantum fields of the theory, namely the pions, but those corresponding to the background connection and to the composite operator implementing the non-linear constraint [1,2]. These amplitudes are named ancestor amplitudes.

At every order in the loop expansion there is only a finite number of divergent ancestor amplitudes. They uniquely fix the divergent amplitudes involving the pions. Moreover, non-renormalizability of this theory in four dimensions can be traced back to the instability of the classical non-linear local symmetry, that gets deformed by quantum corrections. These results hold for the full off-shell amplitudes [3].

A comment is in order here. In Reference [4] it has been argued that Minimal Subtraction is a symmetric scheme, fulfilling all the symmetries of the NLSM in the LFE approach. This in particular entails that all finite parts of the needed higher order counterterms are consistently set to zero. It should be stressed that this is not the most general solution compatible with the symmetries and the WPC, that is commonly used in the spirit of the most popular effective field theory point of view. Indeed, these finite parts are constrained neither by the LFE nor by the WPC and thus, mathematically, they can be freely chosen, as far as they are introduced at the order prescribed by the WPC and without violating the LFE.

The four dimensional SU(2) NLSM provides a relatively simple playground where to test the approach based on the LFE, that can be further generalized to the SU(N) case (and possibly even to a more general Lie group).

Moreover, when the background vector field becomes dynamical, the SU(2) NLSM action allows one to generate a mass term for the gauge field à la Stückelberg [44,45]. The resulting non-linear implementation of the spontaneous symmetry breaking mechanism (as opposed to the linear Higgs mechanism) is widely used in the context of electroweak low energy effective field theories, that are a very important tool in the model-independent analysis of LHC data [46-49]. 


\section{The Classical Non-Linear Sigma Model}

The classical SU(2) NLSM in $D$ dimensions is defined by the action

$$
S_{0}=\int d^{D} x \frac{m_{D}^{2}}{4} \operatorname{Tr}\left(\partial_{\mu} \Omega^{\dagger} \partial^{\mu} \Omega\right)
$$

where the matrix $\Omega$ is a $\mathrm{SU}(2)$ group element given by

$$
\Omega=\frac{1}{m_{D}}\left(\phi_{0}+i \phi_{a} \tau_{a}\right), \quad \Omega^{\dagger} \Omega=1, \quad \operatorname{det} \Omega=1, \quad \phi_{0}^{2}+\phi_{a}^{2}=m_{D}^{2}
$$

In the above equation $\tau_{a}, a=1,2,3$ are the Pauli matrices and $m_{D}=m^{D / 2-1}$ is the mass scale of the theory. $m$ has mass dimension $1 . \phi_{a}$ are the three independent fields parameterizing the matrix $\Omega$, while we choose the positive solution of the non-linear constraint, yielding

$$
\phi_{0}=\sqrt{m_{D}^{2}-\phi_{a}^{2}}
$$

In components one finds

$$
S_{0}=\int d^{D} x\left(\frac{1}{2} \partial_{\mu} \phi_{a} \partial^{\mu} \phi_{a}+\frac{1}{2} \frac{\phi_{a} \partial_{\mu} \phi_{a} \phi_{b} \partial^{\mu} \phi_{b}}{\phi_{0}^{2}}\right)
$$

The model therefore contains non-polynomial, derivative interactions for the massless scalars $\phi_{a}$.

Equation (2) is invariant under a global $\mathrm{SU}(2)_{\mathrm{L}} \times \mathrm{SU}(2)_{\mathrm{R}}$ chiral transformation

$$
\Omega^{\prime}=U \Omega V^{\dagger}, \quad U \in \mathrm{SU}(2)_{\mathrm{L}}, \quad V \in \mathrm{SU}(2)_{\mathrm{R}}
$$

We notice that such a global transformation is non-linearly realized, as can be easily seen by looking at its infinitesimal version. E.g., for the left transformation one finds:

$$
\delta \phi_{a}=\frac{1}{2} \alpha \phi_{0}(x)+\frac{1}{2} \epsilon_{a b c} \phi_{b}(x) \alpha_{c}, \quad \delta \phi_{0}(x)=-\frac{1}{2} \alpha \phi_{a}(x)
$$

Since $\phi_{0}$ is given by Equation (4), the first term in the r.h.s. of $\delta \phi_{a}$ is non-linear (and even non-polynomial) in the quantum fields.

Perturbative quantization of the NLSM requires to carry out the path-integral

$$
Z[J]=\int \mathcal{D} \phi_{a} \exp \left(i S_{0}\left[\phi_{a}\right]+i \int d^{D} x J_{a} \phi_{a}\right)
$$

by expanding around the free theory and by treating the second term in the r.h.s. of Equation (5) as an interaction. Notice that in Equation (8) the sources $J_{a}$ are coupled to the fields $\phi_{a}$ over which the path-integral is performed. In momentum space the propagator for the $\phi_{a}$ fields is

$$
\Delta_{\phi_{a} \phi_{b}}=i \frac{\delta_{a b}}{p^{2}}
$$

The mass dimension of the $\phi_{a}$ is therefore $D / 2-1$, in agreement with Equation (3).

The presence of two derivatives in the interaction term is the cause (in dimensions greater than 2) of severe UV divergences, leading to the non-renormalizability of the theory. 


\section{The Approach based on the Local Functional Equation}

Some years ago it was recognized that the most effective classification of the UV divergences (both for on-shell and off-shell amplitudes) of the NLSM cannot be achieved in terms of the quantized fields $\phi_{a}$, as it usually happens in power-counting renormalizable theories, but rather through the so-called ancestor amplitudes, i.e., the Green's functions of certain composite operators, whose knowledge completely determines the amplitudes involving at least one $\phi_{a}$-leg. This property follows as a consequence of the existence of an additional local functional identity, the so-called Local Functional Equation (LFE) [1].

The LFE stems from the local $\mathrm{SU}(2)_{\mathrm{L}}$-symmetry that can be established from the gauge transformation of the flat connection $F_{\mu}$ associated with the matrix $\Omega$ :

$$
F_{\mu}=i \Omega \partial_{\mu} \Omega^{\dagger}=\frac{1}{2} F_{a \mu} \tau^{a}
$$

i.e., the local $\mathrm{SU}_{\mathrm{L}}(2)$-transformation of $\Omega$

$$
\Omega^{\prime}=U \Omega
$$

induces a gauge transformation of the flat connection, namely

$$
F_{\mu}^{\prime}=U F_{\mu} U^{\dagger}+i U \partial_{\mu} U^{\dagger}
$$

$S_{0}$ in Equation (2) is not invariant under local $\mathrm{SU}(2)_{\mathrm{L}}$ transformations; however it is easy to made it invariant, once one realizes that it can be written as

$$
S_{0}=\int d^{D} x \frac{m_{D}^{2}}{4} \operatorname{Tr}\left(F_{\mu}^{2}\right)
$$

Since $F_{\mu}$ transforms as a gauge connection, one can introduce an additional external classical vector source $\tilde{J}_{\mu}=\frac{1}{2} \tilde{J}_{a \mu} \tau^{a}$ and replace $S_{0}$ with

$$
S=\int d^{D} x \frac{m_{D}^{2}}{4} \operatorname{Tr}\left(F_{\mu}-\tilde{J}_{\mu}\right)^{2}
$$

If one requires that $\tilde{J}_{a \mu}$ transforms as a gauge connection under the local $\mathrm{SU}(2)_{\mathrm{L}}$ group, $S$ in Equation (14) is invariant under a local $\mathrm{SU}(2)_{\mathrm{L}}$ symmetry given by

$$
\begin{aligned}
& \delta \phi_{a}=\frac{1}{2} \alpha_{a} \phi_{0}+\frac{1}{2} \epsilon_{a b c} \phi_{b} \alpha_{c}, \quad \delta \phi_{0}=-\frac{1}{2} \alpha_{a} \phi_{a} \\
& \delta \tilde{J}_{a \mu}=\partial_{\mu} \alpha_{a}+\epsilon_{a b c} \tilde{J}_{b \mu} \alpha_{c}
\end{aligned}
$$

Notice that in the above equation $\alpha_{a}$ is a local parameter.

In order to implement the classical local $\mathrm{SU}(2)_{\mathrm{L}}$ invariance at the quantum level, one needs to define the composite operator $\phi_{0}$ in Equation (4) by coupling it in the classical action to an external source $K_{0}$ through the term

$$
S_{e x t}=\int d^{D} x K_{0} \phi_{0}
$$


$K_{0}$ is invariant under $\delta$.

The important observation now is that the variation of full one-particle irreducible (1-PI) vertex functional $\Gamma^{(0)}=S+S_{\text {ext }}$ is linear in the quantized fields $\phi_{a}$, i.e.,

$$
\delta \Gamma^{(0)}=-\frac{1}{2} \int d^{D} x \alpha_{a}(x) K_{0}(x) \phi_{a}(x)
$$

By taking a derivative of both sides of the above equation w.r.t. $\alpha_{a}(x)$ one obtains the LFE for the tree-level vertex functional $\Gamma^{(0)}$ :

$\mathcal{W}_{a}\left(\Gamma^{(0)}\right)=-\partial_{\mu} \frac{\delta \Gamma^{(0)}}{\delta \tilde{J}_{a \mu}}+\epsilon_{a c b} \tilde{J}_{c \mu} \frac{\delta \Gamma^{(0)}}{\delta \tilde{J}_{b \mu}}+\frac{1}{2} \frac{\delta \Gamma^{(0)}}{\delta K_{0}(x)} \frac{\delta \Gamma^{(0)}}{\delta \phi_{a}(x)}+\frac{1}{2} \epsilon_{a b c} \phi_{c}(x) \frac{\delta \Gamma^{(0)}}{\delta \phi_{b}(x)}=-\frac{1}{2} K_{0}(x) \phi_{a}(x)$

Notice that the $\phi_{0}$-term, entering in the variation of the $\phi_{a}$ field, is generated by $\frac{\delta \Gamma^{(0)}}{\delta K_{0}(x)}$. The advantage of this formulation resides in the fact that it is suitable to be promoted at the quantum level. Indeed by defining the composite operator $\phi_{0}$ by taking functional derivatives w.r.t. its source $K_{0}$, one is able to control its renormalization, once radiative corrections are included [50].

In the following Section we are going to give a compact and self-contained presentation of the algebraic techniques used to deal with bilinear functional equations like the LFE in Equation (18).

\section{Ancestor Amplitudes and the Weak Power-Counting}

We are going to discuss in this Section the consequences of the LFE for the full vertex functional. The imposition of a quantum symmetry in a non-power-counting renormalizable theory is a subtle problem, since in general there is no control on the dimensions of the possible breaking terms as strong as the one guaranteed by the Quantum Action Principle (QAP) in the renormalizable case. Let us discuss the latter case first.

\subsection{Renormalizable Theories and the Quantum Action Principle}

If the tree-level functional $\Gamma^{(0)}$ is power-counting renormalizable, the renormalization procedure [51] provides a way to compute all higher-order terms in the loop expansion of the full vertex functional $\Gamma[\Phi, \chi]=\sum_{n=0}^{\infty} \hbar^{n} \Gamma^{(n)}[\Phi, \chi]$, depending on the set of quantized fields $\Phi$ and external sources collectively denoted by $\chi$, by fixing order by order only a finite set of action-like normalization conditions. One says that the classical action is therefore stable under radiative corrections, namely the number of free parameters does not increase with the loop order.

This procedure is a recursive one, since it allows to construct $\Gamma^{(n)}$ once $\Gamma^{(j)}, j<n$ are known. From a combinatorial point of view, it turns out that $\Gamma$ is the generating functional of the 1-PI renormalized Feynman amplitudes.

A desirable feature of power-counting renormalizable theories is that the dependence of 1-PI Green's functions under an infinitesimal variations of the quantized fields and of the parameters of the model is controlled by the so-called Quantum Action Principle (QAP) [52-55] and can be expressed as the insertion of certain local operators with UV dimensions determined by their tree-level approximation (i.e., a polynomial in the fields, the external sources and derivatives thereof). 
Let us now consider a certain symmetry $\delta$ of the tree-level $\Gamma^{(0)}$ classical action. Under the condition that the symmetry $\delta$ is non-anomalous [56], it can be extended to the full vertex functional $\Gamma$. In many cases of physical interest the proof that the symmetry is non-anomalous can be performed by making use of cohomological tools. Namely one writes the functional equation associated with the $\delta$-invariance of the tree-level vertex functional as follows:

$$
\mathcal{S}\left(\Gamma^{(0)}\right) \equiv \int d^{D} x \sum_{\Phi} \frac{\delta \Gamma^{(0)}}{\delta \Phi(x)} \frac{\delta \Gamma^{(0)}}{\delta \Phi^{*}(x)}=0
$$

where $\Phi^{*}$ is an external source coupled in the tree-level vertex functional to the $\delta$-transformation of $\Phi$ and the sum is over the quantized fields. $\Phi^{*}$ are known as antifields [33]. If $\delta$ is nilpotent (as it happens, e.g., for the Becchi-Rouet-Stora-Tyutin (BRST) operator [57-59] in gauge theories), the recursive proof of the absence of obstructions to the fulfillment of Equation (19) works as follows. Suppose that Equation (19) is satisfied up to order $n-1$ in the loop expansion. Then by the QAP the $n$-th order breaking

$$
\Delta^{(n)} \equiv \int d^{D} x \sum_{\Phi}\left(\frac{\delta \Gamma^{(0)}}{\delta \Phi(x)} \frac{\delta \Gamma^{(n)}}{\delta \Phi^{*}(x)}+\frac{\delta \Gamma^{(n)}}{\delta \Phi(x)} \frac{\delta \Gamma^{(0)}}{\delta \Phi^{*}(x)}+\sum_{j=1}^{n-1} \frac{\delta \Gamma^{(j)}}{\delta \Phi(x)} \frac{\delta \Gamma^{(n-j)}}{\delta \Phi^{*}(x)}\right)
$$

is a polynomial in the fields, the external sources and their derivatives. The term involving $\Gamma^{(n)}$ in Equation (20) allows to define the linearized operator $\mathcal{S}_{0}$ according to

$$
\mathcal{S}_{0}\left(\Gamma^{(n)}\right) \equiv \int d^{D} x \sum_{\Phi}\left(\frac{\delta \Gamma^{(0)}}{\delta \Phi(x)} \frac{\delta \Gamma^{(n)}}{\delta \Phi^{*}(x)}+\frac{\delta \Gamma^{(n)}}{\delta \Phi(x)} \frac{\delta \Gamma^{(0)}}{\delta \Phi^{*}(x)}\right)
$$

$\mathcal{S}_{0}$ is also nilpotent, as a consequence of the nilpotency of $\delta$ and of the tree-level invariance in Equation (19). By exploiting this fact and by applying $\mathcal{S}_{0}$ on both sides of Equation (20) one finds

$$
\mathcal{S}_{0}\left(\Delta^{(n)}\right)=0
$$

provided that the Wess-Zumino consistency condition [60]

$$
\mathcal{S}_{0}\left(\sum_{j=1}^{n-1} \frac{\delta \Gamma^{(j)}}{\delta \Phi(x)} \frac{\delta \Gamma^{(n-j)}}{\delta \Phi^{*}(x)}\right)=0
$$

holds. This is the case, e.g., for the BRST symmetry and the associated master Equation (19), since Equation (23) turns out to be a consequence of a generalized Jacobi identity for the Batalin-Vilkovisky bracket for the conjugated variables $\left(\Phi, \Phi^{*}\right)[33]$.

The problem of establishing whether the functional identity

$$
\mathcal{S}(\Gamma)=0
$$

holds at order $n$ then boils down to prove that the most general solution to Equation (22) is of the form

$$
\Delta^{(n)}=-\mathcal{S}_{0}\left(\Xi^{(n)}\right)
$$

since then $\Gamma^{\prime(n)} \equiv \Gamma^{(n)}+\Xi^{(n)}$ will fulfill Equation (24) at order $n$ in the loop expansion. I.e., the problem reduces to the computation of the cohomology $H\left(\mathcal{S}_{0}\right)$ of the operator $\mathcal{S}_{0}$ in the space of 
integrated local polynomials in the fields, the external sources and their derivatives. Two $\mathcal{S}_{0}$-invariant integrated local polynomials $\mathcal{J}_{1}$ and $\mathcal{J}_{2}$ belong to the same cohomology class in $H\left(\mathcal{S}_{0}\right)$ if and only if

$$
\mathcal{J}_{1}=\mathcal{J}_{2}+\mathcal{S}_{0}(\mathcal{K})
$$

for some integrated local polynomial $\mathcal{K}$. In particular, $H\left(\mathcal{S}_{0}\right)$ is empty if the only cohomology class is the one of the zero element, so that the condition that $\mathcal{J}_{1}$ is $\mathcal{S}_{0}$-invariant implies that

$$
\mathcal{J}_{1}=\mathcal{S}_{0}(\mathcal{K})
$$

for some $\mathcal{K}$. Hence if one can prove that the cohomology of the operator $\mathcal{S}_{0}$ is empty in the space of breaking terms, then Equation (25) must be fulfilled by some choice of the functional $\Xi^{(n)}$. Moreover it must be checked that the UV dimensions of the possible counterterms $\Xi^{(n)}$ are compatible with the action-like condition, so that renormalizability of the theory is not violated. An extensive review of BRST cohomologies for gauge theories is given in [61].

\subsection{Non-Renormalizable Theories}

The QAP does not in general hold for non-renormalizable theories. This does not come as a surprise, since the appearance of UV divergences with higher and higher degree, as one goes up with the loop order, prevents to characterize the induced breaking of a functional identity in terms of a polynomial of a given finite degree (independent of the loop order).

Moreover for the NLSM another important difference must be stressed: the basic Green's functions of the theory are not those of the quantized fields $\phi_{a}$, but those of the flat connection coupled to the external vector source $\tilde{J}_{a \mu}$ and of the non-linear constraint $\phi_{0}$ (coupled to $K_{0}$ ). This result follows from the invertibility of

$$
\frac{\delta \Gamma}{\delta K_{0}}=\phi_{0}+O(\hbar)
$$

as a formal power series in $\hbar$ (since $\left.\phi_{0}\right|_{\phi_{a}=0}=m_{D}$ ). Then the LFE for the vertex functional $\Gamma$

$$
\mathcal{W}_{a}(\Gamma)=-\frac{1}{2} K_{0}(x) \phi_{a}(x)
$$

can be seen as a first-order functional differential equation controlling the dependence of $\Gamma$ on the fields $\phi_{a}$. Provided that a solution exists (as will be proven in Section 5), Equation (28) determines all the amplitudes involving at least one external $\phi_{a}$-leg in terms of the boundary condition provided by the functional $\Gamma\left[\tilde{J}, K_{0}\right]=\left.\Gamma\left[\phi, \tilde{J}, K_{0}\right]\right|_{\phi_{a}=0}$.

$\Gamma\left[\tilde{J}, K_{0}\right]$ is the generating functional of the so called ancestor amplitudes, i.e., the 1-PI amplitudes involving only external $\tilde{J}$ and $K_{0}$ legs.

It is therefore reasonable to assume the LFE in Equation (28) as the starting point for the quantization of the theory.

From a path-integral point of view, Equation (28) implies that one is performing an integration over the SU(2)-invariant Haar measure of the group, namely one is computing

$$
Z\left[J, \tilde{J}_{\mu}, K_{0}\right]=\int \mathcal{D} \Omega(\phi) \exp \left(i \Gamma^{(0)}\left[\phi, \tilde{J}_{\mu}, K_{0}\right]+i \int d^{D} x J_{a} \phi_{a}\right)
$$


where we denote by $\mathcal{D} \Omega(\phi)$ the $\mathrm{SU}(2)$ Haar measure (in the coordinate representation spanned by the fields $\phi_{a}$ ). This clarifies the geometrical meaning of the LFE.

\subsection{Weak Power-Counting}

As we have already noticed, in four dimensions the NLSM is non power-counting renormalizable, since already at one loop level an infinite number of divergent $\phi$-amplitudes exists. One may wonder whether the UV behavior of the ancestor amplitudes (the boundary conditions to the LFE) is better. It turns out that this is indeed the case and one finds that in $D$ dimensions a $n$-th loop Feynman amplitude $\mathcal{G}$ with $N_{K_{0}}$ external $K_{0}$-legs and $N_{\tilde{J}}$ external $\tilde{J}$-legs has superficial degree of divergence given by [2]

$$
d(\mathcal{G}) \leq(D-2) n+2-N_{\tilde{J}}-2 N_{K_{0}}
$$

The proof is straightforward although somehow lengthy and will not be reported here. It can be found in [2]. Equation (30) establishes the Weak Power-Counting (WPC) condition: at every loop order only a finite number of superficially divergent ancestor amplitudes exist.

For instance, in $D=4$ and at one loop order, Equation (30) reduces to

$$
d(\mathcal{G}) \leq 4-N_{\tilde{J}}-2 N_{K_{0}}
$$

i.e., UV divergent amplitudes involve only up to four external $\tilde{J}_{\mu}$ legs or two $K_{0}$-legs.

By taking into account Lorentz-invariance and global $\mathrm{SU}(2)_{\mathrm{R}}$ symmetry, the list of UV divergent amplitudes reduces to

$$
\begin{aligned}
& \int d^{4} x \partial_{\mu} \tilde{J}_{a \nu} \partial^{\mu} \tilde{J}_{a}^{\nu}, \quad \int d^{4} x\left(\partial \tilde{J}_{a}\right)^{2}, \quad \int d^{4} x \epsilon_{a b c} \partial_{\mu} \tilde{J}_{a \nu} \tilde{J}_{b}^{\mu} \tilde{J}_{c}^{\nu}, \quad \int d^{4} x\left(\tilde{J}_{a}\right)^{2}\left(\tilde{J}_{b}\right)^{2} \\
& \int d^{4} x \tilde{J}_{a \mu} \tilde{J}_{b}^{\mu} \tilde{J}_{a \nu} \tilde{J}_{b}^{\nu}, \quad \int d^{4} x \tilde{J}_{a \mu}^{2}, \quad \int d^{4} x K_{0}^{2}, \quad \int d^{4} x K_{0} \tilde{J}_{a}^{2}
\end{aligned}
$$

Notice that the counterterms are local.

It should be emphasized that the model is not power-counting renormalizable, even when ancestor amplitudes are considered, since according to Equation (30) the number of UV divergent amplitudes increases as the loop order $n$ grows.

A special case is the 2-dimensional NLSM. For $D=2$ Equation (30) yields

$$
d(\mathcal{G}) \leq 2-N_{\tilde{J}}-2 N_{K_{0}}
$$

i.e., at every loop order there can be only two UV divergent ancestor amplitudes, namely

$$
\int d^{2} x \tilde{J}^{2} \quad \text { and } \quad \int d^{2} x K_{0}
$$

These are precisely of the same functional form as the ancestor amplitudes entering in the tree-level vertex functional and, in this sense, the model shares the stability property of the classical action typical of power-counting renormalizable models. Renormalizability of the 2-dimensional NLSM can also be established by relying on the Ward identity of global SU(2) symmetry (see e.g., [37]). 
A comment is in order here. In References [24,25] the external fields are the sources of connected Green's functions of certain quark-antiquark currents. The ancestor amplitudes in the NLSM, in the approach based on the LFE, do not have a direct physical interpretation of this type, however they have a very clear geometrical meaning. First of all, $\tilde{J}_{\mu}$ is the source coupled to the flat connection naturally associated with the group element $\Omega$. On the other hand, $K_{0}$ is the unique scalar source required, in the special case of the SU(2) group, in order to control the renormalization of the non-linear classical SU(2) transformation of the $\phi_{a}$ 's and thus plays the role of the so-called antifields [33,50]. The extension to a general Lie group $G$ is addressed at the end of Section 5 .

\section{Cohomological Analysis of the LFE}

In order to study the properties of the LFE, it is very convenient to introduce a fictious BRST operator $s$ by promoting the gauge parameters $\alpha_{a}(x)$ to classical anticommuting ghosts $\omega_{a}(x)$. I.e., one sets

$$
\begin{aligned}
& s \tilde{J}_{a \mu}=\partial_{\mu} \omega_{a}+\epsilon_{a b c} \tilde{J}_{b \mu} \omega_{c}, \quad s \phi_{a}=\frac{1}{2} \omega_{a} \phi_{0}+\frac{1}{2} \epsilon_{a b c} \phi_{b} \omega_{c}, \quad s \phi_{0}=-\frac{1}{2} \omega_{a} \phi_{a} \\
& s K_{0}=\frac{1}{2} \omega_{a} \frac{\delta \Gamma^{(0)}}{\delta \phi_{a}(x)}, \quad s \omega_{a}=-\frac{1}{2} \epsilon_{a b c} \omega_{b} \omega_{c}
\end{aligned}
$$

Some comments are in order here. First of all the BRST operator $s$ acts also on the external source $K_{0}$. Moreover, the BRST transformation of $\omega_{a}$ is fixed by nilpotency, namely $s^{2}=0$.

The introduction of the ghosts allows to define a grading w.r.t. the conserved ghost number. $\omega$ has ghost number +1 , while all the other fields and sources have ghost number zero. (The ghost number was called the Faddeev-Popov ( $\Phi \Pi)$ charge in [2].)

In terms of the operator $s$ we can write the $n$-th order projection $(n \geq 1)$ of the LFE in Equation (28) as follows:

$$
\left[\int d^{D} x \omega_{a} \mathcal{W}_{a}(\Gamma)\right]^{(n)}=s \Gamma^{(n)}+\sum_{j=1}^{n-1} \int d^{D} x \frac{1}{2} \omega_{a} \frac{\delta \Gamma^{(j)}}{\delta K_{0}} \frac{\delta \Gamma^{(n-j)}}{\delta \phi_{a}}=0
$$

Notice that the bilinear term in the LFE manifests itself into the presence of the mixed $\frac{\delta \Gamma^{(j)}}{\delta K_{0}} \frac{\delta \Gamma^{(n-j)}}{\delta \phi_{a}}$ contribution. Moreover in the r.h.s. there is no contribution from the breaking term linear in $\phi_{a}$ in Equation (18) since the latter remains classical.

Suppose now that all divergences have been recursively subtracted up to order $n-1$. At the $n$-th order the UV divergent part can only come from the term involving $\Gamma^{(n)}$ in Equation (35) and therefore, if the LFE holds, one gets a condition on the UV divergent part $\Gamma_{\text {pol }}^{(n)}$ of $\Gamma^{(n)}$ :

$$
s \Gamma_{\text {pol }}^{(n)}=0
$$

To be specific, one can use Dimensional Regularization and subtract only the pole part of the ancestor amplitudes (after the proper normalization of the ancestor background connection amplitudes

$$
\frac{m}{m_{D}} \frac{\delta^{(n)} \Gamma}{\delta \tilde{J}_{a_{1}}^{\mu_{1}} \ldots \delta \tilde{J}_{a_{n}}^{\mu_{n}}}
$$

The LFE then fixes the correct factor for the normalization of amplitudes involving $K_{0}$ ). This subtraction procedure has been shown to be symmetric [2,4], i.e., to preserve the LFE. The pole parts before subtraction obey the condition in Equation (36). 
By the nilpotency of $s$, solving Equation (36) is equivalent to computing the cohomology of the BRST operator $s$ in the space of local functionals in $\tilde{J}, \phi, K_{0}$ and their derivatives with ghost number zero. This can be achieved by using the techniques developed in [62].

One first builds invariant combinations in one-to-one correspondence with the ancestor variables $\tilde{J}_{a \mu}$ and $K_{0}$. For that purpose it is more convenient to switch back to matrix notation. The difference $I_{\mu} \equiv F_{\mu}-\tilde{J}_{\mu}$ transforms in the adjoint representation of $\mathrm{SU}(2)$, being the difference of two gauge connections. Thus the conjugate of such a difference w.r.t. $\Omega$

$$
j_{\mu}=j_{a \mu} \frac{\tau_{a}}{2}=\Omega^{\dagger} I_{\mu} \Omega
$$

is invariant under $s$. By direct computation one finds

$$
\begin{aligned}
m_{D}^{2} j_{a \mu} & =m_{D}^{2} I_{a \mu}-2 \phi_{b}^{2} I_{a \mu}+2 \phi_{b} I_{b \mu} \phi_{a}+2 \phi_{0} \epsilon_{a b c} \phi_{b} I_{c \mu} \\
& \equiv m_{D}^{2} R_{b a} I_{b \mu}
\end{aligned}
$$

The matrix $R_{b a}$ is an element of the adjoint representation of SU(2) and therefore the mapping $\tilde{J}_{a \mu} \rightarrow j_{a \mu}$ is invertible.

One can also prove that the following combination

$$
\bar{K}_{0} \equiv \frac{m_{D}^{2} K_{0}}{\phi_{0}}-\phi_{a} \frac{\delta S}{\delta \phi_{a}}
$$

is invariant [2]. At $\phi_{a}=0$ one gets

$$
\left.\bar{K}_{0}\right|_{\phi_{a}=0}=m_{D} K_{0}
$$

and therefore the transformation $K_{0} \rightarrow \bar{K}_{0}$ is also invertible.

In terms of the new variables $\bar{K}_{0}$ and $j_{\mu}$ and by differentiating Equation (36) w.r.t. $\omega_{a}$ one gets

$$
\Theta_{a b} \frac{\delta \Gamma_{p o l}^{(n)}[j, \bar{K}, \phi]}{\delta \phi_{b}}=0
$$

where $s \phi_{b}=\omega_{a} \Theta_{a b}$, i.e.,

$$
\Theta_{a b}=\frac{1}{2} \phi_{0} \delta_{a b}+\frac{1}{2} \epsilon_{a b c} \phi_{c}
$$

$\Theta_{a b}$ is invertible and thus Equation (41) yields

$$
\frac{\delta \Gamma_{p o l}^{(n)}\left[j, \bar{K}_{0}, \phi\right]}{\delta \phi_{b}}=0
$$

This equation is a very powerful one. It states that the $n$-th order divergences (after the theory has been made finite up to order $n-1$ ) of the $\phi$-fields can only appear through the invariant combinations $\bar{K}_{0}$ and $j_{a \mu}$. These invariant variables have been called bleached variables and they are in one-to-one correspondence with the ancestor variables $K_{0}$ and $\tilde{J}_{a \mu}$. 
The subtraction strategy is thus the following. One computes the divergent part of the properly normalized ancestor amplitudes that are superficially divergent at a given loop order according to the WPC formula in Equation (30). Then the replacement $\tilde{J}_{a \mu} \rightarrow j_{a \mu}$ and $K_{0} \rightarrow \bar{K}_{0}$ is carried out. This gives the full set of counterterms required to make the theory finite at order $n$ in the loop expansion.

As an example, we give here the explicit form of the one-loop divergent counterterms for the NLSM in $D=4$ [2] (notice that we have set $g=1$ according to our conventions in this paper):

$$
\begin{aligned}
\hat{\Gamma}^{(1)}= & \frac{1}{D-4}\left[-\frac{1}{12} \frac{1}{(4 \pi)^{2}} \frac{m_{D}^{2}}{m^{2}}\left(\mathcal{I}_{1}-\mathcal{I}_{2}-\mathcal{I}_{3}\right)+\frac{1}{(4 \pi)^{2}} \frac{1}{48} \frac{m_{D}^{2}}{m^{2}}\left(\mathcal{I}_{6}+2 \mathcal{I}_{7}\right)\right. \\
& \left.+\frac{1}{(4 \pi)^{2}} \frac{3}{2} \frac{1}{m^{2} m_{D}^{2}} \mathcal{I}_{4}+\frac{1}{(4 \pi)^{2}} \frac{1}{2} \frac{1}{m^{2}} \mathcal{I}_{5}\right]
\end{aligned}
$$

By projecting the above equation on the relevant monomial in the $\phi_{a}$ fields one can get the divergences of the descendant amplitudes. As an example, for the four point $\phi_{a}$ function one gets by explicit computation that the contribution from the combination $\mathcal{I}_{1}-\mathcal{I}_{2}-\mathcal{I}_{3}$ is zero, while the remaining invariants give

$$
\begin{aligned}
\hat{\Gamma}^{(1)}[\phi \phi \phi \phi]=- & \frac{1}{D-4} \frac{1}{m_{D}^{2} m^{2}(4 \pi)^{2}} \\
& \int d^{D} x\left(-\frac{1}{3} \partial_{\mu} \phi_{a} \partial^{\mu} \phi_{a} \partial_{\nu} \phi_{b} \partial^{\nu} \phi_{b}-\frac{2}{3} \partial_{\mu} \phi_{a} \partial_{\nu} \phi_{a} \partial^{\mu} \phi_{b} \partial^{\nu} \phi_{b}\right. \\
& \left.-\frac{3}{2} \phi_{a} \square \phi_{a} \phi_{b} \square \phi_{b}-2 \phi_{a} \square \phi_{a} \partial_{\mu} \phi_{b} \partial^{\mu} \phi_{b}\right)
\end{aligned}
$$

The invariants in the combination $\mathcal{I}_{6}+2 \mathcal{I}_{7}$ generate the counterterms in the first line between square brackets; these counterterms are globally SU(2) invariant. The other terms are generated by invariants involving the source $K_{0}$. In [39,40] they were constructed by means of a (non-locally invertible) field redefinition of $\phi_{a}$. The full set of mixed four point amplitudes involving at least one $\phi_{a}$ legs and the external sources $\tilde{J}_{\mu}$ and $K_{0}$ can be found in [2].

The correspondence with the linear sigma model in the large coupling limit has been studied in [5].

The massive NLSM in the LFE formulation has been studied in [15], while the symmetric subtraction procedure for the LFE associated with polar coordinates in the simplest case of the free complex scalar field has been given in [16].

In the SU(2) NLSM just one scalar source $K_{0}$ is sufficient in order to formulate the LFE. For an arbitrary Lie group $G$ the LFE can always be written if one introduces a full set of antifields $\phi_{I}^{*}$, as follows. Let us denote by $\Omega\left(\phi_{I}\right)$ the group element belonging to $G$, parameterized by local coordinates $\phi_{I}$. Then under an infinitesimal left $G$-transformation of parameters $\alpha_{J}$

$$
\delta \Omega=i \alpha_{J} T_{J} \Omega
$$

where $T_{J}$ are the generators of the group $G$, one has

$$
\delta \phi_{I}=S_{I J}(\phi) \alpha_{J}
$$

It is convenient to promote the local left invariance to a BRST symmetry by upgrading the parameters $\alpha_{J}$ to local classical anticommuting ghosts $C_{J}$. Then one can introduce in the usual way the couplings with the antifields $\phi_{I}^{*}$ through

$$
S_{e x t}=\int d^{D} x \phi_{I}^{*} S_{I J}(\phi) C_{J}
$$


and then write the corresponding BV master equation [33]. This is the generalization of the LFE valid for the group $G$. The cohomology of the linearized BV operator (which is the main tool for identifying the bleached variables, as shown above) has been studied for any Lie group $G$ in [62].

\section{Higher Loops}

At orders $n>1$ the LFE for $\Gamma^{(n)}$ is an inhomogeneous equation

$$
s \Gamma^{(n)}=\Delta^{(n)} \equiv-\frac{1}{2} \int d^{D} x \omega_{a} \sum_{j=1}^{n-1} \frac{\delta \Gamma^{(j)}}{\delta K_{0}} \frac{\delta \Gamma^{(n-j)}}{\delta \phi_{a}}
$$

The above equation can be explicitly integrated by using the techniques of the Slavnov-Taylor (ST) parameterization of the effective action [63-65] (originally developed in order to provide a strategy for the restoration of the ST identity of non-anomalous gauge theories in the absence of a symmetric regularization).

For that purpose it is convenient to redefine the ghost according to

$$
\bar{\omega}_{a}=\Theta_{a b} \omega_{b}
$$

where $\Theta_{a b}$ is given in Equation (42). The action of $s$ then reduces to

$$
s \bar{K}_{0}=s j_{a \mu}=0, \quad s \phi_{a}=\bar{\omega}_{a}, \quad s \bar{\omega}_{a}=0
$$

This means that the variables $\bar{K}_{0}$ and $j_{a \mu}$ are invariant, while the pair $\left(\phi_{a}, \bar{\omega}_{a}\right)$ is a BRST doublet (i.e., a pair of variables $u, v$ such that $s u=v, s v=0$ ) [33,66].

By the nilpotency of $s$ the following consistency condition must hold for $\Delta^{(n)}$ :

$$
s \Delta^{(n)}=0
$$

The fulfillment of the above equation as a consequence of the validity of the LFE up to order $n-1$ is proven in [63]. In terms of the new variables Equation (49) reads

$$
\int d^{D} x \bar{\omega}_{a} \frac{\delta \Gamma^{(n)}}{\delta \phi_{a}}=\Delta^{(n)}\left[\bar{\omega}_{a}, \phi_{a}, \bar{K}_{0}, j_{a \mu}\right]
$$

By noticing that $\Delta^{(n)}$ is linear in $\bar{\omega}_{a}$ and by differentiating Equation (53) w.r.t. $\bar{\omega}_{a}$ we arrive at

$$
\frac{\delta \Gamma^{(n)}}{\delta \phi_{a}(x)}=\frac{\delta \Delta^{(n)}}{\delta \bar{\omega}_{a}(x)}
$$

The above equation controls the explicit dependence of the $n$-th order vertex functional on $\phi_{a}$ (there is in addition an implicit dependence on $\phi_{a}$ through the variables $j_{a \mu}$ and $\bar{K}_{0}$ ).

The explicit dependence on $\phi_{a}$ only appears through lower order terms. Hence it does not influence the $n$-th order ancestor amplitudes.

The solution of Equation (49) can be written in compact form by using a homotopy operator. Indeed $\Gamma^{(n)}$ will be the sum of a $n$-th order contribution $\mathcal{A}^{(n)}$, depending only on $j_{a \mu}$ and $\bar{K}_{0}$, plus a lower order term:

$$
\begin{aligned}
\Gamma^{(n)}\left[\phi_{a}, K_{0}, \tilde{J}_{a \mu}\right]= & \mathcal{A}^{(n)}\left[\overline{K_{0}}, j_{a \mu}\right] \\
& +\int d^{D} x \int_{0}^{1} d t \phi_{a}(x) \lambda_{t} \frac{\delta \Delta^{(n)}}{\delta \bar{\omega}_{a}(x)}
\end{aligned}
$$


The operator $\lambda_{t}$ acts as follows on a generic functional $X\left[\phi_{a}, \bar{\omega}_{a}, \bar{K}_{0}, j_{a \mu}\right]$ :

$$
\lambda_{t} X\left[\phi_{a}, \bar{\omega}_{a}, \bar{K}_{0}, j_{a \mu}\right]=X\left[t \phi_{a}, t \bar{\omega}_{a}, \bar{K}_{0}, j_{a \mu}\right]
$$

The homotopy operator $\kappa$ for the BRST differential $s$ in the second line of Equation (55) is therefore given by

$$
\kappa=\int d^{D} x \int_{0}^{1} d t \phi_{a}(x) \lambda_{t} \frac{\delta}{\delta \bar{\omega}_{a}(x)}
$$

and satisfies the condition

$$
\{s, \kappa\}=\mathbf{1}
$$

where 1 denotes the identity on the space of functionals spanned by $\bar{\omega}_{a}, \phi_{a}$.

An important remark is in order here. The theory remains finite and respects the LFE if one adds to $\Gamma^{(n)}$ some integrated local monomials in $j_{a \mu}$ and $\bar{K}_{0}$ and ordinary derivatives thereof (with finite coefficients), compatible with Lorentz symmetry and global SU(2) invariance, while respecting the WPC condition in Equation (30):

$$
\Gamma_{\text {finite }}^{(n)}=\sum_{j} \int d^{D} x \mathcal{M}_{j}\left(j_{a \mu}, \bar{K}_{0}\right)
$$

This is a consequence of the non power-counting renormalizability of the theory: one can introduce order by order in the loop expansion an increasing number of finite parameters that do not appear in the classical action. Notice that they cannot be inserted back at tree-level: if one performs such an operation, the WPC condition is lost.

This observation suggests that these finite parameters cannot be easily understood as physical free parameters of the theory, since they cannot appear in the tree-level action. It was then proposed to define the model by choosing the symmetric subtraction scheme discussed in Section 5 and by considering as physical parameters only those present in the classical action plus the scale of the radiative corrections $\Lambda$ [4]. While acceptable on physical grounds, from the mathematical point of view one may wonder whether there is some deeper reason justifying such a strategy. We will comment briefly on this point in the Conclusions.

\section{Applications to Yang-Mills and the Electroweak Theory}

When the vector source $\tilde{J}_{a \mu}$ becomes a dynamical gauge field, the NLSM action gives rise to the Stückelberg mass term [67].

The subtraction procedure based on the LFE has been used to implement a mathematically consistent formulation of non-linearly realized massive Yang-Mills theory. SU(2) Yang-Mills in the LFE formalism has been formulated in [6]. The pseudo-Goldstone fields take over the role of the $\phi_{a}$ fields of the NLSM. Their Green's functions are fixed by the LFE. The WPC proves to be very restrictive, since by imposing the WPC condition it turns out that the only allowed classical solution is the usual Yang-Mills theory plus the Stückelberg mass term. 
This is a very powerful (and somehow surprising) result. Indeed all possible monomials constructed out of $j_{a \mu}$ and ordinary derivatives thereof are gauge-invariant and therefore they could be used as interaction vertices in the classical action.

Otherwise said, the peculiar structure of the Yang-Mills action

$$
S_{\mathrm{YM}}=-\int d^{4} x \frac{1}{4} G_{a \mu \nu} G_{a}^{\mu \nu}
$$

where $G_{a \mu \nu}$ denotes the field strength of the gauge field $A_{a \mu}$

$$
G_{a \mu \nu}=\partial_{\mu} A_{a \nu}-\partial_{\nu} A_{a \mu}+f_{a b c} A_{b \mu} A_{c \nu}
$$

is not automatically enforced by the requirement of gauge invariance if the gauge group is non-linearly realized. However if the WPC condition is satisfied, the only admissible solution becomes Yang-Mills theory plus the Stückelberg mass term:

$$
S_{\mathrm{nlYM}}=S_{\mathrm{YM}}+\int d^{4} x \frac{M^{2}}{2}\left(A_{a \mu}-F_{a \mu}\right)^{2}
$$

Massive Yang-Mills theory in the presence of a non-linearly realized gauge group is physically unitary [67] (despite the fact that it violates the Froissart bound [68-74] at tree-level). The counterterms in the Landau gauge have been computed at one loop level in [7]. The formulation of the theory in a general 't Hooft gauge has been given in [8].

The approach based on the LFE can also be used for non-perturbative studies of Yang-Mills theory on the lattice. The phase diagram of SU(2) Yang-Mills has been considered in [17]. Emerging evidence is being accumulated about the formation of isospin scalar bound states [18] in the supposedly confined phase of the theory [19].

An analytic approach based on the massless bound-state formalism for the implementation of the Schwinger mechanism in non-Abelian gauge theories has been presented in [75-77].

A very important physical application of non-linearly realized gauge theories is the formulation of a non-linearly realized electroweak theory, based on the group $\mathrm{SU}(2) \times \mathrm{U}(1)$. The set of gauge fields comprises the $\mathrm{SU}(2)$ fields $A_{a \mu}$ and the hypercharge U(1) gauge connection $B_{\mu}$. By using the technique of bleached variables one can first construct SU(2) invariant variables in one-to-one correspondence with $A_{\mu}=A_{a \mu} \frac{\tau_{a}}{2}[8]:$

$$
w_{\mu}=\Omega^{\dagger} g A_{\mu} \Omega-g^{\prime} \frac{\tau_{3}}{2} B_{\mu}+i \Omega^{\dagger} \partial_{\mu} \Omega \equiv w_{a \mu} \frac{\tau_{a}}{2}
$$

In the above equation we have reinserted back for later convenience the $\mathrm{SU}(2)$ and $\mathrm{U}(1)$ coupling constants $g$ and $g^{\prime}$. Since $w_{\mu}$ is $\mathbf{S U}(2)$ invariant, the hypercharge generator coincides with the electric charge generator. $w_{3 \mu}$ is then the bleached counterpart of the $Z_{\mu}$ field, since

$$
Z_{\mu}=\left.\frac{1}{\sqrt{g^{2}+g^{\prime 2}}} w_{3 \mu}\right|_{\phi_{a}=0}=c_{W} A_{3 \mu}-s_{W} B_{\mu}
$$


where $s_{W}$ and $c_{W}$ are the sine and cosine of the Weinberg angle

$$
s_{W}=\frac{g^{\prime}}{\sqrt{g^{2}+g^{\prime 2}}}, \quad c_{W}=\frac{g}{\sqrt{g^{2}+g^{\prime 2}}}
$$

The photon $A_{\mu}$ is described by the combination orthogonal to $Z_{\mu}$, namely

$$
A_{\mu}=s_{W} A_{3 \mu}+c_{W} B_{\mu}
$$

One can built out of $A_{1 \mu}$ and $A_{2 \mu}$ the charged $W^{ \pm}$field

$$
W_{\mu}^{ \pm}=\frac{1}{\sqrt{2}}\left(A_{1 \mu} \mp i A_{2 \mu}\right)
$$

whose bleached counterpart is simply

$$
w_{\mu}^{ \pm}=\frac{1}{\sqrt{2}}\left(w_{1 \mu} \mp i w_{2 \mu}\right)
$$

The WPC allows for the same symmetric couplings of the Standard Model and for two independent mass invariants [9-11]

$$
M_{W}^{2} w^{+} w^{-}+\frac{M_{Z}^{2}}{2} w_{3 \mu}^{2}
$$

where the mass of the $Z$ and $W$ bosons are not related by the Weinberg relation

$$
M_{Z}=\frac{M_{W}}{c_{W}}
$$

This is a peculiar signature of the mass generation mechanism à la Stückelberg, that is not present in the linearly realized theory à la Brout-Englert-Higgs [78-80] (even if one discards the condition of power-counting renormalizability in favour of the WPC) [12].

The inclusion of physical scalar resonances in the non-linearly realized electroweak model, while respecting the WPC, yields some definite prediction for the Beyond the Standard Model (BSM) sector. Indeed it turns out that it is impossible to add a scalar singlet without breaking the WPC condition. The minimal solution requires a SU(2) doublet of scalars, leading to a CP-even physical field (to be identified with the recently discovered scalar resonance at $125.6 \mathrm{GeV}$ ) and to three additional heavier physical states, one CP-odd and neutral and two charged ones [13]. The proof of the WPC in this model and the BRST identification of physical states has been given in [14].

The WPC and the symmetries of the theory select uniquely the tree-level action of the non-linearly realized electroweak model. As in the NLSM case, mathematically additional finite counterterms are allowed at higher orders in the loop expansion. In [4] it has been argued that they cannot be interpreted as additional physical parameters (unlike in the effective field theory approach), on the basis of the observation that they are forbidden at tree-level by the WPC, and this strategy has been consistently applied in $[7,11]$. 
The question remains open of whether a Renormalization Group equation exists, involving a finite change in the higher order subtractions, in such a way to compensate the change in the sliding scale $\Lambda$ of the radiative corrections. We notice that in this case the finite higher order counterterms would be a function of the tree-level parameters only (unlike in the conventional effective field theory approach, where they are treated as independent extra free parameters). This issue deserves further investigation, since obviously the possibility of running the scale $\Lambda$ in a mathematically consistent way would allow to obtain physical predictions of the same observables applicable in different energy regimes.

\section{Conclusions}

The LFE makes it apparent that the independent amplitudes of the NLSM are not those of the quantum fields, over which the path-integral is carried out, but rather those of the background connection $\tilde{J}_{\mu}$ and of the source $K_{0}$, coupled to the solution of the non-linear constraint $\phi_{0}$. The WPC can be formulated only for these ancestor amplitudes; the LFE in turn fixes the descendant amplitudes, involving at least one pion external leg. Within this formulation, the minimal symmetric subtraction discussed in Section 5 is natural, since it provides a way to implement the idea that the number of ancestor interaction vertices, appearing in the classical action and compatible with the WPC, must be finite.

However, it should be stressed that the most general solution to the LFE, compatible with the WPC, does not forbid to choose different finite parts of the higher order symmetric counterterms (as in the most standard view of effective field theories, where such arbitrariness is associated with extra free parameters of the non-renormalizable theory), as far as they are introduced at the order prescribed by the WPC condition and without violating the LFE.

In this connection it should be noticed that the addition of the symmetric finite renormalizations in Equation (59), that are allowed by the symmetries of the theory, is equivalent to a change in the Hopf algebra [81,82] of the model. This is because the finite counterterms in Equation (59) modify the set of 1-PI Feynman diagrams on which the Hopf algebra is constructed, as a dual of the enveloping algebra of the Lie algebra of Feynman graphs. The approach to renormalization based on Hopf algebras is known to be equivalent [83] to the traditional approach based on the Bogoliubov recursive formula and its explicit solution through the Zimmermann's forest formula [84]. For models endowed with a WPC it might provide new insights into the structure of the UV divergences of the theory. This connection seems to deserve further investigations.

\section{Acknowledgements}

It is a pleasure to acknowledge many enlightening discussions with R. Ferrari. Useful comments and a careful reading of the manuscript by $\mathrm{D}$. Bettinelli are also gratefully acknowledged. 


\section{Appendix}

One-Loop Invariants

We report here the invariants controlling the one-loop divergences of the NLSM in $D=4$ [2].

$$
\begin{aligned}
& \mathcal{I}_{1}=\int d^{D} x\left[D_{\mu}(F-\tilde{J})_{\nu}\right]_{a}\left[D^{\mu}(F-\tilde{J})^{\nu}\right]_{a}, \\
& \mathcal{I}_{2}=\int d^{D} x\left[D_{\mu}(F-\tilde{J})^{\mu}\right]_{a}\left[D_{\nu}(F-\tilde{J})^{\nu}\right]_{a}, \\
& \mathcal{I}_{3}=\int d^{D} x \epsilon_{a b c}\left[D_{\mu}(F-\tilde{J})_{\nu}\right]_{a}\left(F_{b}^{\mu}-\tilde{J}_{b}^{\mu}\right)\left(F_{c}^{\nu}-\tilde{J}_{c}^{\nu}\right), \\
& \mathcal{I}_{4}=\int d^{D} x\left(\frac{m_{D}^{2} K_{0}}{\phi_{0}}-\phi_{a} \frac{\delta S}{\delta \phi_{a}}\right)^{2}, \\
& \mathcal{I}_{5}=\int d^{D} x\left(\frac{m_{D}^{2} K_{0}}{\phi_{0}}-\phi_{a} \frac{\delta S}{\delta \phi_{a}}\right)\left(F_{b}^{\mu}-\tilde{J}_{b}^{\mu}\right)^{2}, \\
& \mathcal{I}_{6}=\int d^{D} x\left(F_{a}^{\mu}-\tilde{J}_{a}^{\mu}\right)^{2}\left(F_{b}^{\nu}-\tilde{J}_{b}^{\nu}\right)^{2}, \\
& \mathcal{I}_{7}=\int d^{D} x\left(F_{a}^{\mu}-\tilde{J}_{a}^{\mu}\right)\left(F_{a}^{\nu}-\tilde{J}_{a}^{\nu}\right)\left(F_{b \mu}-\tilde{J}_{b \mu}\right)\left(F_{b \nu}-\tilde{J}_{b \nu}\right)
\end{aligned}
$$

In the above equation $D_{\mu}[F]$ stands for the covariant derivative w.r.t. $F_{a \mu}$

$$
D_{\mu}[F]_{a b}=\delta_{a b} \partial_{\mu}+\epsilon_{a c b} F_{c \mu}
$$

\section{Conflicts of Interest}

The author declares no conflict of interest.

\section{References}

1. Ferrari, R. Endowing the nonlinear sigma model with a flat connection structure: A way to renormalization. JHEP 2005, doi:10.1088/1126-6708/2005/08/048.

2. Ferrari, R.; Quadri, A. A Weak power-counting theorem for the renormalization of the non-linear sigma model in four dimensions. Int. J. Theor. Phys. 2006, 45, 2497-2515.

3. Bettinelli, D.; Ferrari, R.; Quadri, A. Path-integral over non-linearly realized groups and Hierarchy solutions. JHEP 2007, doi:10.1088/1126-6708/2007/03/065.

4. Bettinelli, D.; Ferrari, R.; Quadri, A. Further Comments on the Symmetric Subtraction of the Nonlinear Sigma Model. Int. J. Mod. Phys. 2008, A23, 211-232.

5. Bettinelli, D.; Ferrari, R.; Quadri, A. The Hierarchy principle and the large mass limit of the linear sigma model. Int. J. Theor. Phys. 2007, 46, 2560-2590.

6. Bettinelli, D.; Ferrari, R.; Quadri, A. A Massive Yang-Mills Theory based on the Nonlinearly Realized Gauge Group. Phys. Rev. D 2008, 77, doi:10.1103/PhysRevD.77.045021.

7. Bettinelli, D.; Ferrari, R.; Quadri, A. One-loop self-energy and counterterms in a massive Yang-Mills theory based on the nonlinearly realized gauge group. Phys. Rev. D 2008, 7, doi:10.1103/PhysRevD.77.105012. 
8. Bettinelli, D.; Ferrari, R.; Quadri, A. Gauge Dependence in the Nonlinearly Realized Massive SU(2) Gauge Theory. J. General. Lie Theor. Appl. 2008, 2, 122-126.

9. Bettinelli, D.; Ferrari, R.; Quadri, A. The SU(2) $\times$ U(1) Electroweak Model based on the Nonlinearly Realized Gauge Group. Int. J. Mod. Phys. 2009, A24, 2639-2654.

10. Bettinelli, D.; Ferrari, R.; Quadri, A. The SU(2) $\times$ U(1) Electroweak Model based on the Nonlinearly Realized Gauge Group. II. Functional Equations and the Weak Power-Counting. Acta Phys. Polon. 2010, B41, 597-628.

11. Bettinelli, D.; Ferrari, R.; Quadri, A. One-loop Self-energies in the Electroweak Model with Nonlinearly Realized Gauge Group. Phys. Rev. D 2009, 79, doi:10.1103/PhysRevD.79.125028.

12. Quadri, A. The Algebra of Physical Observables in Nonlinearly Realized Gauge Theories. Eur. Phys. J. 2010, C70, 479-489.

13. Binosi, D.; Quadri, A. Scalar Resonances in the Non-linearly Realized Electroweak Theory. JHEP 2013, 1302, doi:10.1007/JHEP02(2013)020.

14. Bettinelli, D.; Quadri, A. The Stueckelberg Mechanism in the presence of Physical Scalar Resonances. Phys. Rev. D 2013, 88, doi:10.1103/PhysRevD.88.065023.

15. Ferrari, R. A Symmetric Approach to the Massive Nonlinear Sigma Model. J. Math. Phys. 2011, 52, 092303:1-092303:16.

16. Ferrari, R. On the Renormalization of the Complex Scalar Free Field Theory. J. Math. Phys. 2010, 51, 032305:1-032305:20.

17. Ferrari, R. On the Phase Diagram of Massive Yang-Mills. Acta Phys. Polon. 2012, B43, 1965-1980.

18. Ferrari, R. On the Spectrum of Lattice Massive SU(2) YangMills. Acta Phys. Polon. 2013, B44, 1871-1885.

19. Ferrari, R. Metamorphosis versus Decoupling in Nonabelian Gauge Theories at Very High Energies. Acta Phys. Polon. 2012, B43, 1735-1767.

20. Gell-Mann, M.; Levy, M. The axial vector current in beta decay. Nuovo Cim. 1960, 16, 705-726.

21. Weinberg, S. Nonlinear realizations of chiral symmetry. Phys. Rev. 1968, 166, 1568-1577.

22. Coleman, S.R.; Wess, J.; Zumino, B. Structure of phenomenological Lagrangians. 1. Phys. Rev. 1969, 177, 2239-2247.

23. Callan, C.G., Jr.; Coleman, S.R.; Wess, J.; Zumino, B. Structure of phenomenological Lagrangians. 2. Phys. Rev. 1969, 177, 2247-2250.

24. Weinberg, S. Phenomenological Lagrangians. Physica 1979, A96, 327-340.

25. Gasser, J.; Leutwyler, H. Chiral Perturbation Theory to One Loop. Ann. Phys. 1984, 158, $142-210$.

26. Gasser, J.; Leutwyler, H. Chiral Perturbation Theory: Expansions in the Mass of the Strange Quark. Nucl. Phys. B 1985, 250, 465-516.

27. Bijnens, J.; Colangelo, G.; Ecker, G. Renormalization of chiral perturbation theory to order p**6. Ann. Phys. 2000, 280, 100-139.

28. Ecker, G.; Gasser, J.; Leutwyler, H.; Pich, A.; de Rafael, E. Chiral Lagrangians for Massive Spin 1 Fields. Phys. Lett. B 1989, 223, 425-432. 
29. Buchmuller, W.; Wyler, D. Effective Lagrangian Analysis of New Interactions and Flavor Conservation. Nucl. Phys. B 1986, 268, 621-653.

30. Donoghue, J.F. Introduction to the effective field theory description of gravity. Available online: http://arxiv.org/abs/grqc/9512024 (accessed on 15 April 2014).

31. Weinberg, S. The Quantum Theory of Fields. Vol. 2: Modern Applications; Cambridge University Press: Cambridge, UK, 1996.

32. Itzykson, C.; Zuber, J. Quantum Field Theory; McGraw-Hill: New York, NY, USA, 1980.

33. Gomis, J.; Paris, J.; Samuel, S. Antibracket, antifields and gauge theory quantization. Phys. Rep. 1995, 259, 1-145.

34. Gomis, J.; Weinberg, S. Are nonrenormalizable gauge theories renormalizable? Nucl. Phys. B 1996, 469, 473-487.

35. Brezin, E.; Zinn-Justin, J.; Le Guillou, J. Renormalization of the Nonlinear Sigma Model in (Two + Epsilon) Dimension. Phys. Rev. D 1976, 14, 2615-2621.

36. Becchi, C.; Piguet, O. On the Renormalization of Two-dimensional Chiral Models. Nucl. Phys. B 1989, 315, 153-165.

37. Zinn-Justin, J. Quantum Field Theory and Critical Phenomena; International Series of Monographs on Physics; Oxford University Press: Oxford, UK, 2002.

38. Ecker, G.; Honerkamp, J. Application of invariant renormalization to the nonlinear chiral invariant pion lagrangian in the one-loop approximation. Nucl. Phys. B 1971, 35, 481-492.

39. Appelquist, T.; Bernard, C.W. The Nonlinear $\sigma$ Model in the Loop Expansion. Phys. Rev. D 1981, 23, doi:10.1103/PhysRevD.23.425.

40. Tataru, L. One Loop Divergences of the Nonlinear Chiral Theory. Phys. Rev. D 1975, 12, 3351-3352.

41. Gerstein, I.; Jackiw, R.; Weinberg, S.; Lee, B. Chiral loops. Phys. Rev. D 1971, 3, 2486-2492.

42. Charap, J. Closed-loop calculations using a chiral-invariant lagrangian. Phys. Rev. D 1970, 2 , 1554-1561.

43. Honerkamp, J.; Meetz, K. Chiral-invariant perturbation theory. Phys. Rev. D 1971, 3, 1996-1998.

44. Stueckelberg, E. Interaction forces in electrodynamics and in the field theory of nuclear forces. Helv. Phys. Acta 1938, 11, 299-328.

45. Ruegg, H.; Ruiz-Altaba, M. The Stueckelberg field. Int. J. Mod. Phys. 2004, A19, 3265-3348.

46. Altarelli, G.; Mangano, M.L. Electroweak Physics. In Proceedings of CERN Workshop on Standard Model Physics (and More) at the LHC, CERN, Geneva, Switzerland, 25-26 May 1999.

47. Azatov, A.; Contino, R.; Galloway, J. Model-Independent Bounds on a Light Higgs. JHEP 2012, 1204, doi:10.1007/JHEP04(2012)127.

48. Contino, R. The Higgs as a Composite Nambu-Goldstone Boson. Available online: http://arxiv.org/abs/1005.4269 (accessed on 15 April 2014).

49. Espinosa, J.; Grojean, C.; Muhlleitner, M.; Trott, M. First Glimpses at Higgs' face. JHEP 2012 , 1212, doi:DOI:10.1007/JHEP12(2012)045.

50. Zinn-Justin, J. Renormalization of Gauge Theories-Unbroken and broken. Phys. Rev. D 1974, 9, 933-946. 
51. Velo, G.; Wightman, A. Renormalization Theory. In Proceedings of the NATO Advanced Study Institute, Erice, Sicily, Italy, 17-31 August 1975.

52. Breitenlohner, P.; Maison, D. Dimensional Renormalization and the Action Principle. Commun. Math. Phys. 1977, 52, 11-38.

53. Lam, Y.M.P. Perturbation Lagrangian theory for scalar fields: Ward-Takahasi identity and current algebra. Phys. Rev. D 1972, 6, 2145-2161.

54. Lam, Y.M.P. Perturbation lagrangian theory for Dirac fields-Ward-Takahashi identity and current algebra. Phys. Rev. D 1972, 6, 2161-2167.

55. Lowenstein, J. Normal product quantization of currents in Lagrangian field theory. Phys. Rev. D 1971, 4, 2281-2290.

56. Piguet, O.; Sorella, S. Algebraic renormalization: Perturbative renormalization, symmetries and anomalies. Lect. Notes Phys. 1995, M28, 1-134.

57. Becchi, C.; Rouet, A.; Stora, R. Renormalization of Gauge Theories. Ann. Phys. 1976, 98, 287-321.

58. Becchi, C.; Rouet, A.; Stora, R. Renormalization of the Abelian Higgs-Kibble Model. Commun. Math. Phys. 1975, 42, 127-162.

59. Becchi, C.; Rouet, A.; Stora, R. The Abelian Higgs-Kibble Model. Unitarity of the S Operator. Phys. Lett. B 1974, 52, 344-346.

60. Wess, J.; Zumino, B. Consequences of anomalous Ward identities. Phys. Lett. B 1971, 37, 95-97.

61. Barnich, G.; Brandt, F.; Henneaux, M. Local BRST cohomology in gauge theories. Phys. Rep. 2000, 338, 439-569.

62. Henneaux, M.; Wilch, A. Local BRST cohomology of the gauged principal nonlinear sigma model. Phys. Rev. D 1998, 58, 025017:1-025017:14.

63. Quadri, A. Slavnov-Taylor parameterization of Yang-Mills theory with massive fermions in the presence of singlet axial-vector currents. JHEP 2005, 0506, doi:10.1088/1126-6708/ 2005/06/068.

64. Quadri, A. Higher order nonsymmetric counterterms in pure Yang-Mills theory. J. Phys. G 2004, 30, 677-689.

65. Quadri, A. Slavnov-Taylor parameterization for the quantum restoration of BRST symmetries in anomaly free gauge theories. JHEP 2003, 0304, doi:10.1088/1126-6708/2003/04/017.

66. Quadri, A. Algebraic properties of BRST coupled doublets. JHEP 2002, 0205, doi:10.1088/ 1126-6708/2002/05/051.

67. Ferrari, R.; Quadri, A. Physical unitarity for massive non-Abelian gauge theories in the Landau gauge: Stueckelberg and Higgs. JHEP 2004, 0411, doi:10.1088/1126-6708/2004/11/019.

68. Froissart, M. Asymptotic behavior and subtractions in the Mandelstam representation. Phys. Rev. 1961, 123, 1053-1057.

69. Cornwall, J.M.; Levin, D.N.; Tiktopoulos, G. Derivation of Gauge Invariance from High-Energy Unitarity Bounds on the s Matrix. Phys. Rev. D 1974, 10, 1145-1167.

70. Lee, B.W.; Quigg, C.; Thacker, H. Weak Interactions at Very High-Energies: The Role of the Higgs Boson Mass. Phys. Rev. D 1977, 16, 1519-1531. 
71. Weldon, H.A. The Effects of Multiple Higgs Bosons on Tree Unitarity. Phys. Rev. D 1984, 30, 1547-1558.

72. Chanowitz, M.S.; Gaillard, M.K. The TeV Physics of Strongly Interacting W's and Z's. Nucl. Phys. B 1985, 261, 379-431.

73. Gounaris, G.; Kogerler, R.; Neufeld, H. Relationship Between Longitudinally Polarized Vector Bosons and their Unphysical Scalar Partners. Phys. Rev. D 1986, 34, 3257-3259.

74. Bettinelli, D.; Ferrari, R.; Quadri, A. Of Higgs, Unitarity and other Questions. Proc. Steklov Inst. Math. 2011, 272, 22-38.

75. Aguilar, A.; Ibanez, D.; Mathieu, V.; Papavassiliou, J. Massless bound-state excitations and the Schwinger mechanism in QCD. Phys. Rev. D 2012, 85, doi:10.1103/PhysRevD.85.014018 .

76. Aguilar, A.; Binosi, D.; Papavassiliou, J. The dynamical equation of the effective gluon mass. Phys. Rev. D 2011, 84, doi:10.1103/PhysRevD.84.085026.

77. Ibaez, D.; Papavassiliou, J. Gluon mass generation in the massless bound-state formalism. Phys. Rev. D 2013, 87, doi:10.1103/PhysRevD.87.034008.

78. Higgs, P.W. Broken symmetries, massless particles and gauge fields. Phys. Lett. 1964, 12, $132-133$.

79. Higgs, P.W. Broken Symmetries and the Masses of Gauge Bosons. Phys. Rev. Lett. 1964, 13, 508-509.

80. Englert, F.; Brout, R. Broken Symmetry and the Mass of Gauge Vector Mesons. Phys. Rev. Lett. 1964, 13, 321-323.

81. Connes, A.; Kreimer, D. Renormalization in quantum field theory and the Riemann-Hilbert problem. 1. The Hopf algebra structure of graphs and the main theorem. Commun. Math. Phys. 2000, 210, 249-273.

82. Connes, A.; Kreimer, D. Renormalization in quantum field theory and the Riemann-Hilbert problem. 2. The beta function, diffeomorphisms and the renormalization group. Commun. Math. Phys. 2001, 216, 215-241.

83. Ebrahimi-Fard, K.; Patras, F. Exponential renormalization. Ann. Henri Poincare 2010, 11, 943-971.

84. Zimmermann, W. Convergence of Bogolyubov's method of renormalization in momentum space. Commun. Math. Phys. 1969, 15, 208-234.

(c) 2014 by the author; licensee MDPI, Basel, Switzerland. This article is an open access article distributed under the terms and conditions of the Creative Commons Attribution license (http://creativecommons.org/licenses/by/3.0/). 